\title{
Crystallographic Study of Dihydroneopterin Aldolase from Helicobacter pylori
}

\author{
Gary X. Shaw ${ }^{a}$, Scott Cherry, Joseph Tropea, Xinhua Ji ${ }^{b}$ \\ National Cancer Institute, National Institutes of Health, Frederick, MD 21702, USA, \\ ashawg@nih.gov \\ biix@nih.gov
}

Helicobacter pylori infection can lead to stomach cancer and colorectal cancer. The successful cure of $H$. pylori has been reduced by drug resistance. The urgency to develop novel antibiotics against $H$. pylori has fueled continued interest in new targets in the folate biosynthetic pathway, including dihydroneopterin aldolase (DHNA). Our crystallographic study of DHNA from H. pylori (HpDHNA) shows a conserved three-point hydrogen binding motif that is essential for recognizing substrate as seen in DHNAs from other bacteria despite of significant sequence divergence [1-5]. The crystal structure of HpDHNA in complex with a pterin molecule at 1.58-Å resolution reveals a tetrameric form, instead of octameric form as observed in DHNA-ligand complexes from other bacteria. Detailed structural analysis is given to understand the uniqueness of the tetrameric form of the HpDHNA-pterin complex observed in the crystal lattice. The tetrameric form in the crystalline state is consistent with the oligomeric state determined in solution. Analysis of the structure suggests an extended binding groove for additional fragments. Considering the allosteric nature of DHNA active site upon ligand binding [6], fragmentbased method is proposed for the development of larger inhibitors with high potency.

\section{$\underline{\text { References }}$}

[1] Hennig M, D’Arcy A, Hampele IC, Page MG, Oefner C, Dale GE. (1988). Nat Struct Biol., 5:357-362.

[2] Goulding CW, Apostol MI, Sawaya MR, Phillips M, Parseghian A, Eisenberg D (2005). J Mol Biol., 349:61-72.

[3] Garçon A, Levy C, Derrick JP. (2006). J Mol Biol., 360:644-653.

[4] Blaszczyk J, Li Y, Gan J, Yan H, Ji X. (2007), J Mol Biol., 368:161-169.

[5] Blaszczyk J, Lu, Z., Li Y, Yan H, Ji X. (2014), Cell \& Biosci., 4:52-64.

[6] Sanders WJ, Beutel BA. Etc. (2004), J Med Chem., 47:1709-1718. 